\title{
UPDATE Sequencing in prenatal genetics
}

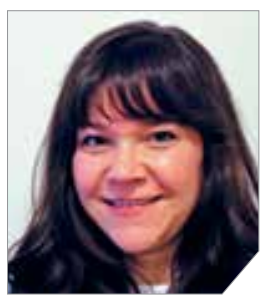

Rebecca Reimers, MD

Dr. Reimers is a Clinical Fellow, Maternal-

Fetal Medicine and Clinical Genetics,

Division of Maternal-Fetal Medicine,

Brigham and Women's Hospital and Boston

Children's Hospital, Boston, Massachusetts.

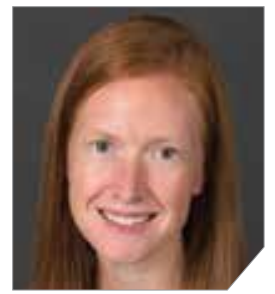

Stephanie Guseh, MD

Dr. Guseh is a Clinical Instructor, Maternal-

Fetal Medicine and Clinical Genetics,

Division of Maternal-Fetal Medicine,

Brigham and Women's Hospital.

The authors report no financial relationships relevant to this article.

Prenatal sequencing studies are providing insights into RASopathy disorders, twin pregnancy genetic changes in embryonic development, and complex genetic alterations within the placenta

\section{IN THIS ARTICLE}

RASopathy panel findings

this page

Sequencing in twin pregnancies

page 17

\section{Genome}

sequencing in

placentas

page 24
P

renatal diagnosis has expanded from identification of aneuploidy to include copy number variants detected on microarray (such as 22q11 deletion syndrome) and now single-gene disorders identified by targeted or exome and genome sequencing. How and when different sequencing tests should be used clinically are questions faced by every provider engaged in modern prenatal diagnosis.

In this Update, we highlight new clinical insights into prenatal sequencing and explore how information gained from sequencing may help us understand some of the unanswered questions in obstetrics.

What is the yield of a RASopathy gene panel with specific prenatal \section{findings?}

Scott A, Di Giosaffatte N, Pinna V, et al. When to test fetuses for RASopathies? Proposition from a systematic analysis of 352 multicenter cases and a postnatal cohort. Genet Med. Published online February 10, 2021. doi:10.1038/s41436-020-01093-7.

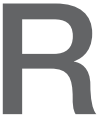

ASopathies, a group of genetic conditions caused by mutations in the RAS/ mitogen-activated protein kinase (RAS-MAPK) pathway, are common, occurring in 1:1,000 to 1:2,500 live births. RASopathies are much more common than 22q11 deletion syndrome, or DiGeorge syndrome, which occurs in 1.4:10,000 live births. ${ }^{1}$
RASopathy disorders include Noonan syndrome, Noonan syndrome with multiple lentigines, Costello syndrome, cardiofaciocuta-

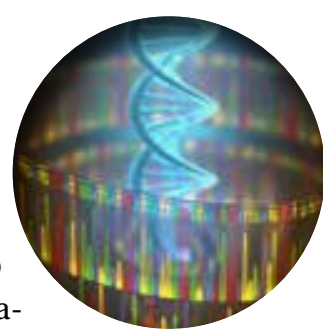
neous syndrome, and Noonan-

like syndrome with loose anagen hair. These are autosomal dominant disorders caused by a pathogenic variant (or mutation) in 1 of more than 20 genes in the RAS-MAPK signaling pathway in the body. Clinical features include congenital anomalies of the kidney and urinary tract, lymphatic anomalies, congenital heart disease (CHD), hypertrophic 


\section{WHAT THIS EVIDENCE MEANS FOR PRACTICE}

Prenatal sequencing panels and exome sequencing are detecting disorders with important implications for prenatal care. If your practice is not testing for RASopathies in prenatal patients with concerning ultrasonography features, you are missing cases. In this study, the most concerning ultrasonography features (more than $20 \%$ diagnosis) were HCM, thoracic effusions and ascites, persistent hydrops, cystic hygroma combined with another suggestive ultrasonography finding, CHD, and persistent cystic hygroma. Isolated ultrasonography findings or findings that resolved had a lower diagnostic yield, and an isolated enlarged NT had a $1 \%$ diagnostic yield, with most cases having an NT larger than $6 \mathrm{~mm}$.

For pretest counseling, in this study $20 \%$ of patients had a variant of uncertain significance, and preparing patients for this possibility is crucial. Most variants of uncertain significance are reclassified to benign when more information is available. Providers can consider sending parental samples concurrently with the fetal sample to help obtain useful information quickly, although the possibility of an inherited pathogenic variant still exists (12\% in this study).

Prenatal diagnosis gives your patients the opportunity to learn about the disorder, plan for treatment and delivery location, and establish their care team before birth or consider pregnancy termination.

\section{FAST}

TRACK

In a cohort study conducted to clarify the utility of testing for RASopathies, among 352 referrals, 50 cases of a RASopathy disorder were diagnosed, and 25 postnatal diagnoses were made after retrospective review cardiomyopathy (HCM), postnatal growth disorders, neurodevelopmental disorders, and more rarely hematologic malignancies. Prenatal clues include an increased nuchal translucency (NT), CHD, cystic hygroma, lymphatic anomalies, anomalies of the kidney and urinary tract, hydrops, and HCM.

\section{Cohort of pregnancies that received a RASopathy panel}

Scott and colleagues sought to clarify the utility of testing for RASopathies with a prenatal gene panel. They conducted a multicenter retrospective cohort study with cases from 2 hospitals in Italy and Canada; data were collected between 2012 and 2019.

Eligible fetuses were those referred to the prenatal genetics clinic because of an increased NT, increased nuchal fold (NF), hydrops, ascites, thoracic effusions, chylothorax, other lymphatic anomalies, CHD, or HCM with a nondiagnostic (negative) microarray or karyotype. All eligible cases had RASopathy molecular testing in the prenatal or neonatal period.
Among the 352 referrals to clinic, 50 cases of a RASopathy disorder were diagnosed. Additionally, to complement this cohort over the same time period, 25 postnatal diagnoses were made after retrospective review performed to ascertain additional prenatal findings. The size of the testing panel ranged from 9 to 20 genes, which were sent to clinical laboratories that performed sequencing based on standard protocols.

\section{Study outcomes}

Overall, $14 \%$ of fetuses with an indication for testing had a pathogenic or likely pathogenic variant (diagnostic) on panel testing among 11 genes (notably, all presented results are after excluding copy number variants and aneuploidy). Fetuses with only 1 ultrasonography finding were much less likely to have a positive result than those with more than 1 ultrasonography finding, $3 \%$ versus $18 \%$. The highest diagnostic yields were for HCM at $69 \%$; thoracic effusions and ascites, $41 \%$; persistent hydrops, $39 \%$; cystic hygroma combined with another suggestive ultrasonography finding, 28\%; CHD, 23\%; and persistent cystic hygroma, $21 \%$. Five fetuses were affected with CHD and HCM, and $44 \%$ had an intrauterine fetal demise.

Importance of NT size. An isolated increased NT had a diagnostic yield of $1 \%$ overall (1/90); however, the size of the NT mattered. Seventeen fetuses had an NT between 3 and $3.5 \mathrm{~mm}$ and none of these had diagnostic sequencing, whereas $26 \%$ with an NT greater than $6 \mathrm{~mm}$ had a diagnostic result (11/43). An increased NF had a diagnostic yield of $25 \%$. Other findings. Of fetuses with a cystic hygroma, $16 \%$ had a pathogenic or likely pathogenic variant, and when these persisted into the second trimester or were associated with other anomalies, the percentages increased to $21 \%$ and $28 \%$, respectively. Of prenatal patients, $20.6 \%$ had variants of uncertain significance, and $12 \%$ of the pathogenic and likely pathogenic variants were inherited, which is less than previously reported series. Additionally, $48 \%$ of the postnatal RASopathy diagnosis group did not have an ultrasonography finding on record review. 


\section{Study strengths and limitations}

This study presents a large cohort of prenatal and neonatal patients tested for RASopathies at 2 international centers with very granular and clinically useful data about ultrasonography findings and yield of panel testing. Prenatal care providers, geneticists, and computational biologists may find this study of great interest and take away useful information and ideas due to the authors' presentation and details.
The number of genes tested changed over the inclusion time period, but this is an inescapable reality of retrospective clinical research in an advancing field. The authors presented the prenatal and postnatal diagnoses ultrasonography findings separately and together. Given the different nature of cohort ascertainment, we prefer to consider these groups separately and have presented the data for the prenatal group.

\section{Sequencing provides insights into twin pregnancies}

Jonsson H, Magnusdottir E, Eggertsson HP, et al. Differences between germline genomes of monozygotic twins. Nat Genet. 2021;53:27-34. doi:10.1038/s41588 -020-00755-1.

Y ou have a monozygotic twin pair with an anomaly and intend to do diagnostic testing for prenatal diagnosis. The question always arises: Do you sample both twins or just one? Surely, they are genetically identical? A wise mentor once instilled a valuable lesson: Monozygotic twins are more likely to have an anomaly. Their existence is already out of the realm of normal. Finally, we now have an engaging and interesting answer to this and other fascinating embryology questions through the work of Jonsson and colleagues.

\section{Study eligibility criteria and treatment protocol}

change that was not present in the

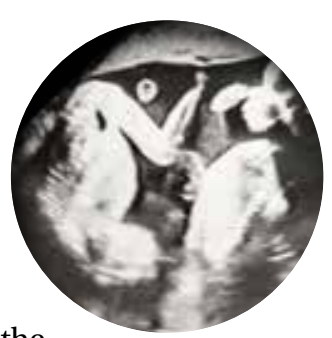
spouse or twin, this genetic change must be present in the oocytes or sperm of the parent twin. The goal of sequencing multiple tissue sources in each twin was to help determine when the genetic change occurred in embryonic development.

\section{Study outcomes}

The authors found that $15 \%$ of twins had mutations that were absent in the other twin. Because of the extent of tissues that had the genetic change, the authors asserted that these changes must have occurred very early in embryonic development (even from one cell after twinning) for the changes to be near-constitutional (among sampled tissues).

An average of 14 genetic differences were found between twin pairs that developed after twinning. However, the number of differences varied. For example, 39 pairs of twins differed by more than 100 changes, and 38 did not differ at all. Differences between twins were more likely in blood samples than in cheek swabs, suggesting that some differences were due to acquired genetic changes in hematologic cell lines, or clonal hematopoiesis. $\overline{\text { FAST }}$

TRACK

The authors found that $15 \%$ of twins had mutations that were absent in the other twin, and these must have occurred very early in embryonic development 


\section{WHAT THIS EVIDENCE MEANS FOR PRACTICE}

We all accumulate changes to our DNA throughout life. The study by Jonsson and colleagues illustrates that for many, this accumulation of genetic changes starts very early in gestation. In the early zygote, the authors observed roughly 1 mutation per cell division prior to the point of twinning. In the realm of prenatal diagnosis, one should consider that monochorionic twins with different phenotypes (that is, an ultrasonography anomaly in 1 of the twin pair) could represent a genetic change rather than an environmental difference. This genetic change may not be shared by the other twin despite originating from the same primordial cell line. The genetic changes that the authors investigated were detected on genome sequencing, which is much more comprehensive than the exome sequencing that is increasingly utilized in rare disease diagnosis. The clinical utility of this observation in prenatal diagnosis has yet to be proven, but this study provides preliminary data that $15 \%$ of monozygotic twins have genetic differences and may warrant individualized testing.

The authors also looked at what percentage of sequenced DNA contained the variants (or mutations) and found that many of these DNA differences were present at high amounts in sequencing reads. This suggests that the DNA changes happened very early after twinning in about one-third of pairs. Additionally, if one twin had a near-constitutional change, in $42 \%$ of pairs the other twin had a different near-constitutional change. Among the triplets, 2 of a triplet pair shared more genetic similarity and were likely descendent from a single split cell and the third likely was formed from a different set of cells.

By examining the offspring of twins,
Jonsson and colleagues found that there were 2.6 early embryonic mutations, and this did not differ when blood or buccal DNA was compared. The rate of transmission of a variant to offspring was proportional to the variant allele frequency (proportion of alternate alleles) in the blood or buccal cells. This is an important counseling point when considering patients with mosaic genetic disorders and counseling about the likelihood of inheritance or transmission to future offspring. If the rate of mosaicism was higher in blood or buccal cells, the likelihood of transmission was higher. Additionally, the mutations did not differ by sex, and there was no relationship to whether the chromosome was maternally or paternally inherited.

\section{Study strengths and limitations}

The authors did not have access to information about chorionicity of the monozygotic twin pairs. Consequently, they were unable to correlate chorionicity with the degree of noted genetic difference between the monozygotic twin pairs. Additionally, although the authors were thoughtful in their utilization of offspring and spouses to infer germline genomic content, the study had a limited number of tissues sampled, which could reduce the applicability. However, the sample size, clinically accessible tissue sampling, and thoughtful analysis used in this study make it an interesting and relevant contribution to reproductive medicine and evolutionary biology.

\section{The genetic landscape of the placenta}

Coorens TH, Oliver TR, Sanghvi R, et al. Inherent mosaicism and extensive mutation of human placentas. Nature. Published online March 10, 2021. doi:10.1038/ s41586-021-03345-1.

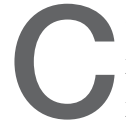
onfined placental mosaicism (CPM) is a phenomenon in which the genetics of the placenta are different from those of the fetus. Historically, this phenomenon has been described in $1 \%$ to $2 \%$ of pregnancies based on karyotype data obtained

CONTINUED ON PAGE 24 


\section{WHAT THIS EVIDENCE MEANS FOR PRACTICE}

With a new appreciation for the smaller genetic alterations that exist within placental tissue, it appears that the rate of CPM has been vastly underestimated. We know that aneuploid placental cells increase the risk of adverse pregnancy outcomes and we may learn more about the contribution of copy number variants and single-nucleotide changes to preeclampsia, growth restriction, and pregnancy loss. Furthermore, as the applications of cell-free fetal DNA (cffDNA) in genetic screening continue to expand, we must exercise caution in assuming that copy number variants or single-nucleotide changes detected by cffDNA reflect those of the developing fetus.

$\overline{\text { FAST }}$

TRACK

The authors

described the

placenta as a

"patchwork" of

independent

genetic units

resulting from

clonal expansion

at different stages

of embryonic

development from chorionic villus sampling. Some studies have demonstrated adverse pregnancy outcomes in the setting of CPM, thought to be secondary to aneuploid cells in the placenta leading to insufficiency or dysfunction.

Although our sophistication and level of detail in prenatal genetic testing has rapidly expanded to include information about copy number variants and singlenucleotide changes, their contribution to CPM has been understudied. Coorens and colleagues recently published a landmark study that describes a surprisingly high rate of mosaicism for these smaller genetic changes.

\section{A cohort study of placentas}

The authors performed whole genome sequencing on placental samples obtained from 37 term pregnancies. Umbilical cord tissue and maternal blood also were collected and served as controls for fetal and maternal genetic profiles, respectively.

In a subgroup of 5 placentas, lasercapture microscopy was used to separate placental cells of different origins, including trophoblastic cells, mesenchymal core cells, and cells originating from the inner cell mass. To investigate variation within different geographic regions of a single placenta, these cell lines were derived multiple times from each quadrant of the 5 placentas.

\section{Placental biopsies revealed "bottlenecks" of genetic differentiation}

Genome sequencing was used uniquely in this study to help delineate the phylogeny of placental cells by tracking somatic mutations both in different geographic locations of each placenta and between different cells of origin within 1 placenta.

The authors concluded that bottlenecks of differentiation in placental development led to unique genetic signatures in every bulk placental sample studied. Their findings led them to describe the placenta as a "patchwork" of independent genetic units resulting from clonal expansion at different stages of embryonic development.

\section{Early insights into human placental cells}

This study provides fascinating insight into the surprisingly high rates of copy number variants and single-gene changes that exist, in mosaic form, within human placentas. The authors distinguish the placenta from other human organs (such as the colon, endometrium, liver, and skin) in which many fewer genetic changes exist. In fact, they suggest parallels between the "mutational signature" of the placenta with rapidly dividing neoplastic cells.

As one of the first investigations into the variation and complexity of genetic changes within the placenta, this study was not designed to draw conclusions regarding the clinical impact of the numerous genetic changes described. Further studies will elucidate the potential contribution of genetically mosaic placentas to common adverse obstetric outcomes. 